\title{
O mix público-privado no Sistema de Saúde Brasileiro: financiamento, oferta e utilização de serviços de saúde
}

\author{
The public-private mix in the Brazilian Health System: \\ financing, delivery and utilization of health services
}

I sabela Soares Santos ${ }^{1}$

M aria Alicia Dominguez Ugá ${ }^{1}$

Silvia M arta Porto ${ }^{1}$

${ }^{1}$ Escola Nacional deSaúde Pública, Fiocruz. Av. Leopoldo Bulhões 1480/

$724, M$ anguinhos. 21041-210 Rio de Janeiro RJ. isantos@ensp.fiocruz.br
Abstract This paper analyzes the public-private mix in the Brazilian H ealth System from the perspective of health care delivery, utilization and financing. M oreover, this quantitativestudy based on secondary data from official databases contemplates the subsidies granted by the government to the private sector. It shows the existence of some inequalities favoring the population having private health plans, a result of the peculiar participation of the privatesector in the Brazilian $\mathrm{H}$ ealth System not only offering supplementary care but duplicating the coverage offered by the public system (called SUS). The analysis is made on the basis of the classification of public-private mix in $\mathrm{H}$ ealth Systems developed by the OECD in 2004, that helps understanding the kinds of inequalities occurring in each type of public-private mix. The inequalities that occur in the Brazilian system must be understood as the result of the duplicated coverage offered by the private market and of the weak public funding for the SUS while granting important subsidies to the private sector.

Key words Public-private mix, Brazilian health system, Duplicated coverage, Supplementary healthcare, Financing, U tilization of health care
Resumo 0 artigo analisa o mix público-privado do sistema de saúde brasileiro a partir da oferta, utilização e financiamento dos serviços de saúde. Contempla os subsídios públicos para o setor privado. Trata-se de um estudo quantitativo, baseado em dados secundários provenientes de bases de dados oficiais. M ostra que existem desigualdades na oferta e na utilização de serviços em prol da população com plano desaúde, em decorrência da peculiar inserção do setor suplementar, que oferece cobertura suplementar e duplicada ao sistema público (SUS), sem desconsiderar que outros fatores podem determinar o uso de serviços desaúde eaumentar as desigualdades. A análiseéfeita com base na tipologia de mix público-privado desenvolvida pela OECD em 2004, que auxilia a compreensão das desi gualdades que ocorrem em cada tipo de mix, emostra que as que ocorrem no sistema de saúde brasilei ro se dão pelo fato de a cobertura de serviços ofertados pelo segmento de seguro privado ser duplicada à cobertura de serviços do SUS. Ainda, as desigualdades verificadas no sistema de saúde brasileiro ocorrem num sistema de saúde em que o financiamento público ao SUS é minoritário e existem grandes subsídios públicos para o setor privado.

Palavras-chave Mix público-privado, Sistema desaúde, Cobertura duplicada, Seguro suplementar, Financiamento, U tilização de serviços 
Introdução

A discussão sobre os modelos de sistemas de saúde vem ganhando novos contornos, após as diversas reformas que, desdeos anos noventa, vêm sendo implementadas tanto nos países europeus como nos países periféricos. Estas, embora com objetivos formais e formatos finais distintos, derivaram, todas, do tratamento do gasto em programas sociais (entre os quais, o da saúde) como instrumento do ajuste macroeconômico, como mostrou Ugá1. Elas resultaram na introdução de mudanças marginais nos sistemas de saúde welfarianos e, diferentemente, no caso dos países latino-americanos, na formatação de sistemas em muitos casos totalmente distintos dos previamente existentes (como é o caso da Colômbia e do M éxico, por exemplo).

$\mathrm{Na}$ maior parte dos casos, o que se observa atualmente é o distanciamento dos modelos tidos como "tipos ideais" (no sentido weberiano) de sistemas de saúde. Não existem hoje - com a exceção de pouquíssimos países - sistemas que se enquadrem no formato "puro" de um dos três tipos clássicos, isto é, desistema nacional de saúde (com prestação exclusivamente estatal efinanciamento exclusivamente por tributos), de sistema pertencente ao seguro social (com financiamento exclusivamente efetuado por meio decontribuições sobre folha de salário) e de sistema de saúde liberal (baseado apenas nas forças de mercado). Existem, sim, sistemas de saúde mistos, que mesclam elementos da esfera pública e da privada, tanto no âmbito da prestação de serviços, como no do financiamento dos mesmos.

Deve-se observar, entretanto, que o formato final desses sistemas resulta da própria história da construção dos mesmos. No que diz respeito ao caso brasileiro, o processo iniciado pelo movimento da reforma sanitária, que culminou com as teses defendidas eaprovadasnaVIII a Conferência Nacional deSaúde, de1986, resultou, na Constituição Federal de 1988, na opção por um modelo inspirado nos sistemas nacionais de saúde de acesso universal eintegral. Entretanto, na realidade, já existia um setor privado robusto, tanto no campo da prestação de serviços ( com um parque hospitalar predominantemente privado), como no quetange ao asseguramento privado (por meio de seguradoras e operadoras de planos privados de saúde, que detêm nada menos que 45,9 miIhões de clientes (março de 2007) ${ }^{2}$.

Este artigo tem por objetivo analisar o formato peculiar do sistema de saúde brasileiro, fundado como sistema nacional de saúde que, entretanto, apresenta uma estrutura fortemente moldada na participação do setor público e do privado, herdada do modelo anterior.

\section{M arco conceitual}

É particularmente útil para a compreensão do nosso sistema de saúde o marco conceitual desenvolvido pela O rganização de Cooperação para o Desenvolvimento Econômico (OECD), em $2004^{3-5}$ e sustentado por diversos autores ${ }^{6-9}$, que diz respeito à forma em que se insere 0 seguro privado no sistema de saúde: suplementar, substitutivo, complementar ou primário.

No primeiro caso, o seguro privado comercializa planos de saúde que oferecem serviços já cobertos pelo sistema público de cunho universal ou pelo seguro social de adesão compulsória. 0 que caracteriza essa forma de inserção do seguro privado é, portanto, a cobertura duplicada de serviços de saúde que dele decorre. Isso não impede que, em alguns casos, o seguro privado suplementar ofereça al guns el ementos adicionais, tais como diferenciação na hotelaria, livre escoIha de prestador e/ou acesso mais ágil que no sistema estatutário. Ele também pode eventualmente prover alguns serviços não cobertos pelo sistema estatutário e que não são considerados como fundamentais para a saúde - por exemplo, cirurgia estética. 0 seguro suplementar está presente em diversos países, tais como Reino Unido, Irlanda, Finlândia, Portugal, Espanha, ItáliaeGrécia.

Nos casos em queo indivíduo deveoptar pelo sistema público ou pelo seguro privado, esteatua como substitutivo. Ele se verifica em países com sistema de seguro social no formato posterior às reformas dos noventa, tais como Alemanha, Holanda, Bélgica e, na América Latina, o Chile.

Ainda, o seguro privado pode ser complementar ao sistema estatutário, quando as pessoas o adquirem para complementar 0 acesso a serviços que não são cobertos pelo sistema estatutário, ou o são apenas parcialmente. N este último caso, 0 seguro privado complementar se destina a cobrir co-pagamentos impostos pelo sistema estatutário, como ocorre na França, por exemplo.

O seguro privado atua como sistema primário quando ele é o elemento preponderante do sistema de saúde, como no caso estadunidense.

N os casos onde o segmento de planos e seguros de saúde é suplementar (e, portanto, associado a uma duplicação de cobertura), importantes problemas são introduzidos, tanto no que diz 
respeito a desigualdades no acesso a serviços de saúde ${ }^{10,11}$, como ao gasto (tendo em vista que, nesses casos, o gasto público não é inferior, em termos de porcentagem do PIB, ao dos países nos quais o seguro privado está ausente), como mostram os dados da OECD ${ }^{12}$. Efetivamente, essa publicação evidencia que, por um lado, os detentores de esquemas privados de asseguramento continuam utilizando serviços públicoseque, por outro, o seguro privado tem freqüentementeadicionado gasto à despesa total em saúde (e não substituído o financiamento público). Além disso, esse segmento econômico vem recebendo, em grande parte dos países, subsídios referentes à dedução dos gastos em seguros de saúde no imposto de renda devido, como acontece também no nosso país.

Não é fortuito que, na formulação da Constituição Federal brasileira de 1988, houvesse tantos embates em relação à denominação do segmento de planos privados e seguros de saúde no Brasil. Com base na tipologia da OECD ${ }^{13}$, a definição de suplementar confirma o caráter incremental do seguro em relação ao sistema nacional de saúde brasileiro constituído pelo Sistema Único de Saúde (SUS), cujo acesso é universal e integral. Entretanto, uma vez consagrado que 0 segmento de planos e seguros de saúde oferece serviços paralelos aos do SUS - diferenciados apenas em termos dehotelaria e, no que tange ao acesso, da maior facilidade para agendar 0 atendimento, da maior possibilidade de escolher medicamentos não genéricos -, verifica-se que, além da função de suplementar aos serviços do SUS, o nosso segmento de seguros privados também é duplicado quanto à cobertura de serviços de saúde.

Como se tentará demonstrar ao longo deste artigo, a construção do sistema nacional de saúde brasileiro a partir de uma base fortemente privatizada - queera a existenteem 1988 - resultou em um modelo absolutamente peculiar, no qual há uma imbricação do estatal e do privado nos principais elementos estruturantes do sistema.

Este artigo analisa o mix público-privado do sistema de saúde brasileiro a partir da oferta, utilização deserviços desaúdee do financiamento dos mesmos, contemplando, ainda, os subsídios que se dão entre o Estado e o setor privado no âmbito do setor saúde.

\section{M etodologia}

Trata-se de um estudo de natureza quantitativa, baseado em dados secundários provenientes de distintas bases de dados oficiais, que analisa 0 mix público-privado do sistema de saúde brasileiro pela oferta, uso e seu financiamento. Também foram identificados subsídios cruzados do Estado para o setor privado, que complementam a análise.

Assim, a oferta foi analisada pelas informações da Pesquisa Assistência M édico-Sanitária (AM S) do IBGE de 2005, cujos dados estão disponíveis no site do IBGE. Foram observadas as características dos estabelecimentos de saúde e de seus equipamentos segundo a natureza jurídica dos mesmos - pública ou privada - e sua relação com o SUS.

Considerou-se como oferta pública os leitos e equipamentos de propriedade estatal e, ainda, aqueles privados contratados e conveniados ao SUS. No que diz respeito à oferta privada, foi considerada exclusivamente a oferta dos serviços em estabelecimentos privados que não estivessem contratados ou conveniados ao SUS.

Para a análise da oferta pública de serviços de saúde, foram excluídas as informações relativas aos estabelecimentos públicos que atendem demandas de clientelas fechadas e, portanto, não são de acesso universal.

Esta análise toma como base populacional a estimada pelo IBGE para o ano 2005, a partir do censo (183.798.218 habitantes) ${ }^{14}$ e assume que a população com plano de saúde coberta em relação a serviços médico-hospitalares, tendo sido excluídas as pessoas que possuem planos de saúde exclusivamente odontológicos, éa informada pelaAN S para dezembro daquele ano (35.151.348 pessoas) ${ }^{15}$.

As informaç̃̃es sobre o uso de serviços segundo fonte definanciamento provêm da metodologia desenvolvida por Porto et al. em 2006 ${ }^{16}$, que tomou como fonte as informações da Pesquisa $\mathrm{N}$ acional por Amostra de Domicílios (PNAD) do IBGE de 2003. Esta análise considerou três variáveis para dimensionar o volume e tipo de serviços que as pessoas possuidoras de plano de saúde utilizaram no SUS.

A primeira variável diz respeito à posse de plano de saúde. A PN AD considera plano público os serviços e planos de saúde mantidos pelo Estado para clientelas fechadas (como forças armadas, por exemplo). Mas estes são tratados neste trabalho como privados, tendo em vista que 0 acesso aos serviços érestrito às respectivas 
clientelas desses planos. Dessa forma, considerou-se como população com plano de saúde o somatório de pessoas que responderam ter plano público ou privado na PNAD.

A segunda variável se refereao tipo de serviço de saúde utilizado. A PNAD pergunta se o respondente recebeu algum atendimento nas duas semanas anteriores à entrevista e sefoi internado no ano anterior. Foram considerados os atendimentos e as internações, por tipo. Note-se que 0 número de internações referido nos atendimentos difere do número de internações apresentado no item específico, devido às diferenças na base temporal de observações.

A terceira variável diz respeito à fonte de financiamento dos serviços de saúde utilizados, se pelo SUS, se pelo plano ou se pelo gasto privado direto. Como as respostas não são excludentes, optou-se pela metodologia desenvolvida por Porto et ${ }^{1} .^{16}$ para que as respostas sobre a fonte de financiamento do serviço de saúde possam somar $100 \%$.

As informações sobre o financiamento dos serviços de saúde referem-seà fonte de financiamento do serviço utilizado informado pela PNAD.

No que concerne aos subsídios do Estado para o setor privado, foram utilizadas estimativas da Secretaria da Receita Federal do M inisté rio da Fazenda ${ }^{17}$ para os gastos governamentais tributários indiretos, a partir das desonerações tributárias para o setor saúde em 2006.

\section{Resultados}

\section{Oferta de serviços de saúde}

O ponto central desta seção éo de identificar as desigualdades na oferta existentes em um sistema de saúde com cobertura e prestação duplicadas, como no caso brasileiro. Para isso, são apresentadas comparações entre a rede de serviços ofertados pel o SU S e os disponibilizados para 0 atendimento dos clientes de planos e seguros privados de saúde. D eve-se destacar que a fonte de informação utilizada apresenta limitações importantes que já foram apontadas na análise efetuada por Viacava e Bahia ${ }^{18}$ em relação à AM S/ IBGE de 1999, equesemantêm na de 2005. Como afirmam os autores, a pesquisa exclui os consultórios médicos particulares que representam a principal oferta ambulatorial dos planos e seguros privados de saúde. Tampouco é possível estimar qual é a parcela exata da oferta de proprie- dade privada prestadora de serviços concomitantemente ao SU S e aos planos eseguros de saúde, que se destina a cada clientela. Ainda assim, é possível efetuar importantes considerações em relação à oferta disponível de serviços de saúde no Brasil.

A maior parte da oferta de serviços hospitalares é de propriedade privada: $62 \%$ dos estabelecimentos com internação e $68 \%$ dos leitos do país. Uma concentração ainda maior observa-se em relação às unidades prestadoras de serviços deapoio diagnóstico eterapêutico - SADT (92\%). Contrariamente, a maior parte das unidades ambulatoriais (78\%) é de propriedade estatal. Cabe lembrar que não foram contabilizados os consultórios médicos privados.

Esses dados se referem apenas à distribuição da oferta segundo a natureza jurídica do prestador, mas não refletem a distribuição entre a oferta disponibilizada para usuários do SU S e os beneficiários dos planos e seguros de saúde. Como na maioria dos países ondea maior parte da oferta éde propriedade privada, o setor público contrata parte desses serviços privados. Segundo a AM S/IBGE de 2005, no Brasil, 69\% dos estabelecimentos privados que contam com internação e $65 \%$ dos leitos privados são contratados pelo SUS. Entretanto, como foi mencionado, esses estabelecimentos também estão contratados pelos planos e seguros privados de saúde.

Contudo, com o objetivo de inferir desigualdades na disponibilidade de oferta entre usuários do SUS e beneficiários de planos e seguros privados de saúde, assumiu-se como oferta pública os leitos e equipamentos de propriedade estatal e, ainda, aqueles privados contratados e conveniados pelo SUS. Por sua vez, a oferta privada considerada é exclusi vamente aquela de estabelecimentos privados não conveniados ou contratados pelo SUS.

Sabe-se que estas definições implicam uma superestimativa da oferta pública e uma subestimação da oferta privada, tendo em vista que os estabel ecimentos privados que oferecem leitos ao SUS também o fazem para os planos e seguros de saúde, bem como para a demanda efetivada pelo pagamento direto. Além disso, muitos leitos que oficialmente são reservados ao SUS podem, na prática, ser utilizados para pacientes privados.

A partir dessas definições, a oferta (superestimada) de leitos SUS seria de 1,81 leitos/1.000 habitantes e a oferta (subestimada) para clientes de planos e seguros privados de saúde, 2,90 leitos/1.000. Destaca-se ainda que, mesmo se se considerasse como população coberta pelo SUS 
exclusivamente a não coberta por planos e seguros privados de saúde, a oferta de leitos SUS (2,24 leitos/1.000 habitantes) seria inferior à estimada para a esfera privada. Este cálculo, embora inadequado do ponto de vista do direito universal à saúde inerente à cidadania, é aqui apresentado apenas com o intuito de evidenciar o grau de desigual dades na oferta de serviços entre o SUS e o segmento suplementar.

Analisando a oferta de equipamentos ediscriminando a oferta SU S e privada da mesma forma efetuada no caso de oferta de leitos, observa-se que, excetuando hemodiálises, a oferta disponível para clientes de planos e seguros de saúde ésignificativamente maior que a estimada como disponibilizada pelo SUS no que se refere a todos os equipamentos de média e al ta complexidade/al to custo (mamógrafo, litotripsor, ultra-som, tomógrafo computadorizado, ressonância magnética, radioterapia, medicina nuclear e raio- $X$ para hemodinâmica), como se observa na Tabela 1.

Tais dados evidenciam, portanto, que no sistema de cobertura duplicada existente no nosso país, o segmento suplementar disponibiliza uma oferta de serviços hospitalares e, principalmente, de equipamentos de alta e média complexidade muito maior do que a observada no SUS.

Tabela 1. Número de equipamentos de média e alta complexidade/alto custo por 100.000 habitantes, segundo SUS e não-SUS. Brasil, 2005.

\begin{tabular}{lcc}
\hline \multicolumn{1}{c}{$\begin{array}{c}\text { Equipamentos } \\
\text { (por 100.000 habitantes) }\end{array}$} & SUS & Privado \\
\hline Mamógrafo & 0,912 & 4,461 \\
Litotripsor & 0,163 & 0,654 \\
Ultra-sonografia & 3,891 & 20,170 \\
Tomógrafo computadorizado & 0,595 & 2,466 \\
Ressonância magnética & 0,131 & 0,879 \\
Radioterapia & 0,168 & 0,270 \\
Medicina nuclear & 0,084 & 0,131 \\
Raio-X para hemodinâmica & 0,190 & 0,532 \\
Hemodiálise & 7,401 & 2,475 \\
\hline
\end{tabular}

Fonte: IBGE, AM S/2005.
Uso de serviços de saúde segundo fonte de financiamento

Segundo a PNAD 2003, 24,6\% da população brasileira tinham plano de saúde naquele ano. Essa cobertura duplicada introduz importantes desigual dades na utilização de serviços, como se observa a seguir. A Tabela 2 evidencia que a população que tem plano de saúde usa mais serviços de saúde que a população brasileira como um todo. 0 percentual de pessoas que internaram no ano anterior à entrevista foi maior entre os que têm plano de saúde (8,3\%) que para toda a população brasileira $(7,0 \%)$ e muito superior à taxa de internações pelo SU S (4,7\%). No que diz respeito aos atendimentos, o percentual foi de 19,7\% para os que tinham plano de saúde, de $14,2 \%$ para toda a população brasileira e $8,1 \%$ para os financiados pelo SUS.

Ainda, evidencia a tabela que $1,3 \%$ da população que possui plano de saúde realizou internações financiadas pelo SUS ou, em outras palavras, que $15,4 \%$ da população possuidora de planos de saúde internada o foram pelo SUS.

Em Porto et al. ${ }^{16}$, evidenciou-se, ainda, que:

a) Entre as pessoas internadas em 2003 que responderam qual a fonte de financiamento da internação, 69,9\% afirmaram têla sido pelo SUS, $24,8 \%$, por meio de planos e seguros de saúde e, finalmente, $5,3 \%$, mediante desembolso direto.

b) Entretanto, existem fortes interseções na utilização de serviços entre o SUS e o segmento da população com planos de saúde. Assim, entre as pessoas que tinham plano, $6,6 \%$ foram internadas pelo próprio plano, 1,3\% foram internadas pelo SU S e $0,3 \%$, mediante gasto privado direto.

c) Considerados exclusivamente os pacientes possuidores de plano de saúde que foram internados em 2003, verificou-se que apenas 79,3\% tiveram a internação financiada pelo próprio plano, sendo o restante internado pelo SUS $(15,4 \%)$ ou por meio de pagamento direto (3,5\%).

No que diz respeito aos atendimentos no ano de $2003,61,3 \%$ foram declarados como realizados pelo SUS. Os planos e seguros de saúde financiaram 27,9\% desses atendimentos, e, ainda, $10,8 \%$ dos mesmos foram financiados por meio de pagamento direto ${ }^{16}$.

Da mesma forma que para as internações, também em relação aos atendimentos, se observa importantes interseções entre o SU S e o segmento da saúde suplementar: a Tabela 4 mostra que $2,3 \%$ das pessoas que possuíam plano de saúde em 2003 foram atendidas pelo SUS, o que 
Tabela 2. População internada e atendida e o financiamento da utilização. Brasil, 2003.

\begin{tabular}{lccc}
\hline & Número & $\begin{array}{c}\% \text { da população } \\
\text { brasileira }(*)\end{array}$ & $\begin{array}{c}\% \text { da população } \\
\text { com plano }(* *)\end{array}$ \\
\hline Internações & & & $\mathrm{n} / \mathrm{a}$ \\
$\quad$ Total de pessoas internadas & 12.332 .546 & $7,0 \%$ & $\mathrm{n} / \mathrm{a}$ \\
Pessoas internadas financiadas pelo SUS & 8.272 .846 & $4,7 \%$ & $\mathrm{n}, 3 \%$ \\
Pessoas com plano de saúde internadas & 3.584 .231 & $\mathrm{n} / \mathrm{a}$ & $1,3 \%$ \\
Pessoas com plano de saúde internadas financiadas & 553.389 & $\mathrm{n} / \mathrm{a}$ & \\
pelo SUS & & & $\mathrm{n} / \mathrm{a}$ \\
Atendimentos & & & $\mathrm{n} / \mathrm{a}$ \\
Total de pessoas que foram atendidas & 24.979 .475 & $14,2 \%$ & $19,7 \%$ \\
Pessoas que foram atendidas financiadas pelo SUS & 14.260 .670 & $8,1 \%$ & $2,3 \%$ \\
Pessoas com plano que foram atendidas & 8.520 .502 & $\mathrm{n} / \mathrm{a}$ & $\mathrm{n} / \mathrm{a}$ \\
Pessoas com plano que foram atendidas financiadas & 1.005 .386 & & \\
pelo SUS & & &
\end{tabular}

Fonte: elaboração própria a partir dos microdados da PNAD/IBGE-2003.

(*) População estimada em base à expansão da amostra a PN AD/IBGE-2003 (175.987.612 habitantes); (**) População estimada em base à expansão da amostra da PN AD/IBGE-2003 (43.215.760 pessoas com planos de saúde) .

Tabela 3. Internações do SUS, segundo tipo de serviço, por posse de plano de saúde do usuário. Brasil, 2003.

\begin{tabular}{lccr}
\hline \multicolumn{1}{c}{$\begin{array}{c}\text { Internações realizadas pelo SUS } \\
\text { (no ano anterior à entrevista) }\end{array}$} & $\begin{array}{c}\text { Total de população } \\
\text { internada pelo SUS }\end{array}$ & $\begin{array}{c}\text { Total de pessoas com plano } \\
\text { de saúde internadas pelo SUS }\end{array}$ & $\%$ \\
\hline Internações para tratamento clínico & 4.831 .192 & 291.574 & 6,04 \\
Internações para parto normal & 988.510 & 59.107 & 5,98 \\
Internações para parto cesáreo & 480.791 & 35.367 & 7,36 \\
Internações para cirurgia & 1.610 .367 & 144.608 & 8,98 \\
Internações para tratamento psiquiátrico & 144.029 & 6.050 & 4,20 \\
Internações para exames & 217.957 & 16.683 & 7,65 \\
Total pessoas de internadas pelo SUS & 8.272 .846 & 553.389 & 6,69 \\
\% de pessoas com plano internadas pelo SUS & & $1,30 \%$ & \\
\hline
\end{tabular}

Fonte: Elaboração própria a partir dos microdados da PN AD/IBGE-2003.

Tabela 4. Atendimentos do SUS, segundo tipo de serviço, por posse de plano de saúde do usuário. Brasil, 2003.

\begin{tabular}{lccc}
\hline \multicolumn{1}{c}{$\begin{array}{c}\text { Atendimentos realizados pelo SUS } \\
\text { (nas duas semanas anteriores à entrevista) }\end{array}$} & $\begin{array}{c}\text { Total de população } \\
\text { atendida pelo SUS }\end{array}$ & $\begin{array}{c}\text { Total de pessoas com } \\
\text { plano de saúde } \\
\text { atendidas pelo SUS }\end{array}$ & $\%$ \\
\hline Consulta médica & 10.858 .188 & 709.294 & 6,53 \\
Consulta odontológica & 623.028 & 46.413 & 7,45 \\
Consulta AC ou parteira & 20.099 & 1.308 & 6,51 \\
Consulta outro profissional de saúde & 192.060 & 16.825 & 8,76 \\
Consulta farmácia & 14.697 & 1.648 & 11,21 \\
Hemodiálise, quimio, radio, ou hemoterapia & 71.331 & 8.253 & 11,57 \\
Vacinação, injeção, curativo, pronto atendimento & 837.548 & 92.190 & 11,01 \\
Cirurgia em ambulatório & 114.143 & 8.173 & 7,16 \\
Gesso ou imobilização & 138.033 & 11.852 & 8,59 \\
Internação hospitalar & 328.431 & 17.065 & 5,20 \\
Exames complementares & 835.440 & 70.700 & 8,46 \\
Outros & 227.672 & 21.665 & 9,52 \\
Total de pessoas atendidas pelo SUS & 14.260 .670 & 1.005 .386 & $\mathbf{7 , 0 5}$ \\
\% de pessoas com plano atendidas pelo SUS & & $2,30 \%$ &
\end{tabular}

Fonte: Elaboração própria a partir dos microdados da PNAD/IBGE-2003. 
corresponde ao fato de que $11,8 \%$ das pessoas com plano atendidas o foram pelo SUS.

Note-se que, se considerados exclusivamente os pacientes com plano de saúde que foram atendidos, apenas $75,9 \%$ tiveram 0 atendimento financiado pelo próprio plano, enquanto $11,8 \%$ foram atendidos pelo SUS e 8,4\% pagaram diretamente. 0 restante $(3,9 \%)$ não respondeu quem financiou 0 atendimento ${ }^{16}$.

Serviços do SUS mais utilizados por pacientes que possuem planos de saúde

A análise da utilização de serviços do SUS mostra que, entre os pacientes internados pelo SUS, 6,7\% possuem planos de saúde privados, como mostram as Tabelas 3 e 4 . Entre os atendimentos prestados pelo SUS, 7,1\% o foram para pacientes com planos.

No que diz respeito às internações, a utilização do SUS por pacientes com plano se destaca em relação às internações cirúrgicas, pois 9,0\% das mesmas foram efetuadas para pacientes com cobertura duplicada.

Este percentual se eleva quando se trata de procedimentos de alto custo/complexidade, tais como hemodiálise, quimio, rádio e hemoterapia, dos quais $11,6 \%$ foram realizados para pacientes com plano de saúde. Da mesma forma, entre alguns serviços em que o SUS se destaca, como vacinações e pronto atendimento, $11,0 \%$ das pessoas que usaram são pacientes possuidores de planos privados.

Subsídios do Estado para o setor privado no âmbito da saúde

Diversos subsídios se dão entre o Estado e o setor privado, no âmbito da saúde. Entre os principais, consta o "gasto tributário", isto é os recursos tributários que o Estado deixa de arrecadar, mediante as desonerações fiscais. Eles atuam como incentivo fiscal aos prestadores e operadoras de planos de saúde filantrópicos e, também, como indutores à compra de serviços e de planos privados, uma vez que o gasto deles provenienteé deduzido da base sobre a qual écalculado o imposto de renda devido por pessoas físicas e jurídicas.

Assim, por um lado, as instituições filantrópicas (queenvolvem tanto prestadores como operadoras de planos de saúde) são isentas dos tributos federais, estaduais e municipais. Segundo U gá et al. ${ }^{19}$, existiam 1.917 hospitaisfilantrópicosno Brasil em 2005. Ainda, segundo a ANS, em março de
2007, existiam 106 operadoras filantrópicas registradas na A gência, com 1.300 .914 beneficiários.

Entidade Filantrópica éaquel a entidade beneficentedeassistênciasocial (definida originalmente na Lei Orgânica deAssistência Social, Lei no 8.742 de 08/12/1993, Art. 30, como "aquelas que prestam, sem fins lucrativos, atendimento e assessoramento aos beneficiários abrangidos por esta lei, bem como atuam na defesa e garantia de seus direitos") e/ou de educação e/ou de saúde, sem fins lucrativos (definida na Lei no 9.718 de 27/11/ 1998, Art. 10, § 3o, sobre a Legislação Tributária Federal, como sendo "a entidade que não apresenta superávit em suas contas ou, caso apresente em determinado exercício, destine o referido resultado integral mente à manuten ção e ao desenvolvimento de seus objetivos sociais"), que cumprecom as exigências da Resolução CNAS 32/99. A concessão ou renovação do Certificado de Filantropia está regulamentada pela Lei Orgânica deAssistência Social, Lei n ${ }^{\circ} .742 / 93$, pelo Decreto 2.536 de 06/04/98 e pela Resolução CN AS 32/99.

Segundo essa Resolução, a concessão ou renovação do Certificado de Filantropia a esse tipo de entidades privadas, sem fins lucrativos, exige que a entidade solicitante comprove as seguintes exigências cumulativas e existentes nos três anos anteriores à concessão ou renovação: (1) estar legalmente constituída no país eem funcionamento; (2) possuir inscrição em um Conselho M unicipal de Assistência Social (CM AS), ou um Conselho Estadual de Assistência Social (CEAS) ou no Consel ho de Assistência Social do Distrito $\mathrm{Fe}$ deral (CASDF); (3) possuir registro no CNAS; e (4) incluir no seu estatuto as condições de a) aplicar suas rendas integral mente no território nacional ena manutenção de seus objetivos institucionais; b) aplicar as subvenções e doações nas finalidades a que está vinculada; c) não distribuir resultados; d) não perceber seus diretores; e) destinar seu patrimônio, em ato constitutivo, ao CNAS ou entidade pública, em caso de dissolução ou extinção; f) não constituir patrimônio de indivíduo ou de sociedade sem caráter beneficente de assistência social; g) prestar serviços gratuitos permanentes e sem qualquer discriminação declientela; eh) aplicar anual menteem gratuidade pelo menos $20 \%$ da receita bruta proveniente da venda de serviços, mais aplicações financeiras, locação de bens e venda de bens não integrantes do ativo imobilizado, mais doações, cujo montante nunca será inferior à isen ção de contribuições sociais usufruída. No caso de entidade de saúde, deverá comprovar anualmente o percentual deatendimentos ao SUS depelo menos $60 \%{ }^{20}$. 
Segundo a Receita Federal, a renúncia fiscal advinda das desonerações tributárias federais usufruídas pelas entidades filantrópicas prevista para 0 ano de 2007 é de nada menos que $R \$ 1,6$ bilhões. A esta cifra deve ser acrescido o montante referente à renúncia fiscal dos demais entes federativos.

Por outro lado, a renúncia fiscal referente às deduções de gastos em saúde sobre a base sobre a qual é calculado o imposto de renda devido se dá em relação às pessoas físicas (que descontam do cálculo da receita tributável os gastos em serviços e em planos de saúde) e às empresas que, ao imputarem como custos os gastos em serviços e planos de saúde dos seus funcionários, diminuem também a base de cálculo do imposto de renda.

Dessa forma, embora não se possa afirmar que a totalidade dos gastos privados em saúde seja financiada pelo Estado pelo gasto tributário (ou renúncia fiscal), uma boa parteo é, na medida em que da base sobre a qual é calculado o Imposto de Renda são deduzidos os gastos privados em serviços e planos de saúde.

A esse respeito, o mesmo documento da Receita Federal prevê que, em 2007, estas deduções tributárias al cançarão o montante de R $\$ 4,1$ biIhões, o que corresponde a $10,1 \%$ do gasto federal em saúde, se considerado o informado pela Sub-Secretaria de Planejamento eO rçamento do M inistério da Saúde ${ }^{21}$.

Outro importante elemento da relação entre Estado e mercado se dá na relação entre o SUS, as empresas que operam planos de saúde e os prestadores de serviços privados.

No quetange às operadoras de planos de saúde, os dados da PN AD/IBGE-2003 mostram que 15,4\% das pessoas que têm plano de saúde privado e foram internadas o foram pelo SUS. Essa porcentagem, no caso dos atendimentos, é de $11,8 \%$. Háalgunsanos, a Lei no 9.656 de 1998 , no seu Artigo 32, instituiu o ressarcimento ao SUS dos serviços por ele prestados a pessoas com asseguramento privado, por uma tabela específica de preços, denominada TUNEP (Tabela Única $\mathrm{N}$ acional de Equivalência de Procedimentos). Entretanto, segundo dados da AN S, desde o final de 1999 até meados de 2006, das 371.761 internações identificadas como aptas para cobrança, apenas 60.586 foram efetivamente pagas pelas seguradoras, ou seja, desde quando foi instituído o ressarcimento até quando foram processadas as informações ${ }^{22}$. 0 pagamento dessas internações pelos valores da TU NEP no período acima referido corresponderam a $\mathrm{R} \$$
76.928.764,00, valor pouco significativo quando comparado com os valores da renúncia fiscal (que somam, na previsão efetuada apenas para 0 ano de 2007, $R \$ 5,7$ bilhões). Processos longos, recursos à justiça por motivos diversos (quando se trata de pacientes detentores de "planos antigos", prévios à regulamentação da Lei n 9.656 , por exemplo) fazem com que esse ressarcimento seja muito pouco expressivo.

No que se refere aos subsídios cruzados entre - SUS eos prestadores privados, deve-semencionar a "dupla porta de entrada" de hospitais privados que também atendem a pacientes do SUS e muitas vezes estabelecem, nessa duplicidade, uma relação promíscua. Como mostra uma pesquisa realizada recentemente por U gá et al. ${ }^{23}$, nada menos que $72 \%$ dos prestadores hospitalares que atuam na saúde suplementar também prestam serviços ao SUS. Em outra pesquisa qualitativa realizada junto a ben eficiários de planos de saúde de entidades hospitalares filantrópicas que possuem operadora própria ${ }^{24}$, verificou-se que serviços não cobertos pelo plano são prestados pelo mesmo estabelecimento com financiamento SUS, o qual, por outro lado, favorece 0 acesso desses beneficiários ao SUS. Assim, como apontam os autores: a relação dos planos com o SU S émarcada pela interdependência quese apresenta pelo encaminhamento para o SUS em virtude de uma restrição que consta na cláusula do plano, do favorecimento de acesso ao SUS pelos usuários e da indistinção entre serviços prestados pelo SU S e pelo plano.

\section{Consideraçõesfinais}

Este trabalho evidenciou fatores cruciais para a compreensão do nosso sistema de saúde que, contando com um segmento de seguradoras e operadoras de planos de saúde suplementar, contém um importante segmento populacional que conta com cobertura duplicada de serviços de saúde.

Assim, em decorrência da peculiar inserção do setor de planos e seguros de saúde no Brasil, que oferecem cobertura suplementar e duplicada, são extremamente importantes as desigualdades observadas na oferta e utilização de serviços a favor da população com plano de saúde: 0 segmento suplementar disponibiliza uma oferta deserviçoshospitalarese, principalmente, de equipamentos de alta complexidade muito superior à do SUS.

Por sua vez, a utilização de serviços de saúde (internações e atendimentos), correspondentementeàs diferençasna oferta, émuito maior, tam- 
bém, na população detentora de planos de saúde, provavelmente devido, adicionalmente, a diferenças existentes em outros fatores determinantes do uso de serviços, tais como níveis de educação e renda. Vale reiterar que $15,4 \%$ da população com plano internada e $11,8 \%$ da população com plano que teve atendimentos utilizou o SUS.

Outro resultado da história da constituição do sistema de saúde brasileiro diz respeito ao fato de que, apesar de tratar-se de um sistema nacional de saúde, ele conta com um financiamento que é minoritariamente $(43,8 \%)$ públi$\mathrm{CO}^{25}$ e uma prestação predominantemente privada, na qual $68 \%$ dos leitos e $92 \%$ dos prestadores de SADT são privados.

O predomínio dos prestadores privados introduz, obviamente, importantes fluxos financeiros da esfera pública para a privada. Ainda, nesse mix existem relações pouco claras entre 0 papel que um mesmo prestador privado exerce em segmentos diferentes (no SUS e no setor de saúde suplementar).
Pouco conhecido é, ademais, o volume de re cursos aportado pelo Estado para planos de clientelas fechadas constituídas de servidores públicos e, por outro lado, a oferta de leitos de hospitais públicos destinada a planos de saúde privados (como, por exemplo, os oferecidos pelo Instituto do Coração, em São Paulo, e do Hospital das Clínicas da Faculdade de M edicina da USP).

Trata-se de um sistema de saúde que, embora constitucionalmente definido como universal e integral, tem o setor privado prestador sustentado pela compra estatal de serviços e um segmento de seguradoras e operadoras de planos de saúde fortemente subsidiado pelo Estado, pelos distintos instrumentos de renúncia fiscal acima apontados.

É hora, portanto, de superar a herança do quefoi construído no período devigência do "corporativismo bifronte e segmentário" 26 dos regimes burocrático-autoritários, em que as políticas sociais se constituem em espaços deinterpenetração do Estado e do setor privado, onde este tem um lugar privilegiado na formulação de políticas.

\section{Colaboradores}

IS Santos, M AD U gá eSM Porto participaram da concepção, do marco teórico, da metodologia, das análises dos resultados e da redação do texto. 0 processamento dos dados foi realizado por IS Santos. 
1. Ugá MAD. Propostas de reforma do setor saúde nos marcos do ajuste macroeconômico [tese]. Rio de Janeiro (RJ): IM S/UERJ; 1997.

2. Agência Nacional de Saúde Suplementar. Caderno de Informação da Saúde Suplementar da ANS: beneficiáriOS, operadoras e planos. Rio de Janeiro: ANS; 2007.

3. Organisation for Economic Co-Operation and Development. Proposal for a Taxonomy of Health Insurance. OECD Study on Private Health Insurance. OECD Health Project: June 2004. [acessado 2007 Mai 15]. Disponível em: http://www.oecd.org/ health/insurance

4. Organisation for Economic Co-Operation and Development. Private $\mathrm{H}$ ealth Insurance in OECD Countries [Policy Brief]. OECD Observer: September 2004. [acessado 2007 M ai 15]. Disponível em: http:/ /www.oecd.org/health/insurance

5. Organisation for Economic Co-Operation and Development. Private $\mathrm{H}$ ealth Insurance in OECD Countries. The OECD Health Project. [acessado $2007 \mathrm{Mai}$ 15]. Disponível em: http://www.oecd.org/health/ insurance

6. Couffinhal A. Concurrence en assurance santé: entre efficacité et selection - Competition in health insurance: between efficiency and selection [Ph.D. dissertation]. Paris: Université Paris IX-Dauphine; 1999.

7. Colombo F, Tapay N. Private Health Insurance in OECD Countries: the Benefits and Costs for Individuals and Health Systems. Paris: OECD; 2004. [OECD Health Working Paper n. 15]

8. Mossialos E, Thompson SM S. Voluntary Health Insurance in Europe Union. Copenhagen: World Health Organization, European Observatory on $\mathrm{H}$ ealth Systems and Policies; 2004.

9. Thompson SM S, Mossialos E. Regulating private health insurance in the European Union: the implications of single market legislation and competition policy. London: LSE Health; 2006. [Working Paper n. 4]

10. Rodríguez M, Stoyanova A. The effect of private insurance access on the choice of GP/Specialist and public/private provider in Spain. Health Economics 2004; 13:689-703.

11. van Doorslaer $E$, Koolman X, Puffer F. Equity in the use of physician visits in OECD countries: has equal treatment for equal need been achieved? In: OECD. $M$ easuring up: improving health systems performance in OECD countries. Paris: OECD; 2002. p. 225-248.

12. Organisation for Economic Co-Operation and Development. Private $\mathrm{H}$ ealth Insurance in OECD Countries. The OECD H ealth Project. [acessado $2007 \mathrm{M}$ ai 15]. Disponível em: http://www.oecd.org/health/ insurance

13. Organisation for Economic Co-Operation and Development. Private $\mathrm{H}$ ealth Insurance in OECD Countries. The OECD H ealth Project. [acessado $2007 \mathrm{M}$ ai 15]. Disponível em: http://www.oecd.org/health/ insurance
14. Instituto Brasileiro de Geografia e Estatística. Censos demográficos e contagem populacional; para os anos intercensitários, estimativas preliminares dos totais populacionais, estratificadas por idade e sexo pelo M S/SE/ Datasus. Rio de Janeiro: IBGE. [acessado $2007 \mathrm{M}$ ai Disponível em: http://w3.datasus.gov.br/datasus/ datasus. php?area $=359$ A 1B379C 6D 0E 0F 359G 23H 0I 1] d6L26M ON \&VInclude=../site/infsaude.php

15. Agência Nacional de Saúde Suplementar. Caderno de Informação em Saúde Suplementar da ANS: beneficiários, operadoras e planos. Rio de Janeiro: ANS; 2007.

16. Porto $S M$, Santos IS, U gá MAD. A utilização de serviços de saúde por sistema de financiamento. Cien Saude Colet 2006; 11(4):895-910.

17. Brasil. M inistério da Fazenda. Secretaria da Receita Federal, Coordenação-Geral de Política Tributária. Demonstrativo dos Gastos Governamentais Indiretos de Natureza Tributária (Gastos Tributários). Brasília: M F/SRF/CGPT; 2007.

18. Viacava F, Bahia L. Oferta de serviços de saúde: uma análise da Pesquisa Assistência M édico-Sanitária (AMS) de 1999. Brasília: IPEA; 2002. [Texto para discussão no 915]

19. Ugá MAD, Barbosa PR, Lemos SML, Vasconcellos M M, Gerschman S, Portela M C. Dimensionamento dos planos de saúde comercializados por hospitais fiIantrópicos no Brasil. Rio de Janeiro: ENSP/Fiocruz; 2004. [acessado 2005 Dez 28]. Disponível em: http:/ /www.ans.gov.br/portal/site/Biblioteca/biblioteca_ topico_17702.asp

20. Portela M C, Lemos S, Barbosa PR, M urat M, U gá MAD, Gerschman S. Caracterização assistencial hospitalar filantrópica no Brasil. Rev. Saúde Pública 2004; 38(6):811-818.

21. Carvalho G. Gasto com Saúde no Brasil em 2006. [acessado $2007 \mathrm{M}$ ai 10]. Disponível em: http:// www.idisa.org.br/site/paginas/visualiza subcategoria. php?sub=324

22. Agência Nacional de Saúde Suplementar. Caderno de Informação de Ressarcimento e Integração com 0 SUS. Rio de Janeiro: ANS; 2006.

23. Ugá MAD, Lemos $S$, Portela $M$, Vasconcellos $M$, Gerschman S. Estudo dos prestadores hospitalares frente às práticas de micro-regulação das operadoras de planos de saúde [relatório de pesquisa]. Rio de Janeiro: ENSP/Fiocruz; 2007. [M imeo]

24. Gerschman $S$, Veiga L, Guimarães $C$, U gá $M A D$, Portela M C Murat M, Barbosa P, Lemos S. Estudo de satisfação dos beneficiários de planos de saúde de hospitais filantrópicos. Cien Saude Colet 2007; 12(2):487-500.

25. Ugá MAD, Santos IS. An analysis of equity in Brazilian health system financing. Health Affairs 2007; 26(4):1017-1028.

26. O'Donnell G. Sobre o corporativismo e a questão do Estado [Cadernos DCP]. Belo Horizonte: UFM G; 1986.

Artigo apresentado em 23/07/2007

Aprovado em 01/10/2007

Versão final apresentada em 23/10/2007 